\title{
The pseudoscalar hadronic channel contribution of the light-by-light process to the muon (g-2) $\mu$ within the nonlocal chiral quark model
}

\author{
A.E. Dorokhov ${ }^{1}$, A.E. Radzhabov ${ }^{2}$, and A.S. Zhevlakov ${ }^{2}$ \\ 1 Bogoliubov Laboratory of Theoretical Physics, JINR, 141980 Dubna, Russia \\ 2 Institute for System Dynamics and Control Theory, 664033 Irkutsk, Russia \\ the date of receipt and acceptance should be inserted later
}

\begin{abstract}
The light-by-light contribution to the anomalous magnetic moment of muon $(g-2)_{\mu}$ from the hadronic exchanges in the neutral pseudoscalar meson channel is calculated in the nonlocal chiral quark model. The full kinematic dependence of the meson-two-photon vertices from the virtualities of the mesons and photons is taken into account. The status of various phenomenological and QCD shortdistance constraints is discussed and the comparison with the predictions of other models is performed. It is demonstrated that the effect of the full kinematic dependence in the meson-photon vertices is to reduce the contribution of pseudoscalar exchages to $a_{\mu}^{\mathrm{PS}, \mathrm{LbL}}$ by approximately factor 1.5 in comparison with the most of previous estimates.
\end{abstract}

\section{Introduction}

The study of the anomalous magnetic moments (AMM) of leptons, $a=(g-2) / 2$, has played an important role in the development of the standard model. At the present level of accuracy, the muon AMM, $a_{\mu}$, leads to the sensitivity to effects of new physics, with particles in the mass range of a few hundred $\mathrm{GeV} / \mathrm{c}^{2}$.

Comparison of the experimental measurements of the muon AMM [1] with the predictions of the standard model (see, for example [2]) shows deviation by approximately $3 \sigma$. The uncertainties of the standard model value for $a_{\mu}$ are dominated by the uncertainties of the hadronic contributions $a_{\mu}^{\text {Strong }}$, since their evaluation involves methods of nonperturbative QCD at large distances. The contribution to the muon AMM to leading order in the QED coupling constant $\alpha$ comes from the hadronic vacuum polarization and the next-to-leading corrections consist of the contributions which are the iteration of the leading order term plus the contribution from the light-by-light (LbL) scattering process. The absolute values of the leading and next-toleading terms are differed by one order of magnitude, but the theoretical accuracy of their extraction is comparable and dominates the overall theoretical error of the standard model result. The hadronic vacuum polarization contribution to the muon AMM is known with accuracy better than one percent owing to the phenomenological analysis of the inclusive $e^{+} e^{-} \rightarrow$ hadrons and $\tau \rightarrow$ hadrons data 3. This, however, is not the case for the LbL contribution known from various QCD motivated approaches with accuracy of order $50 \%$. It is the LbL contribution, which will dominate the theoretical error of the standard model pre- diction in the near future, and more realistic approaches are needed for a better understanding.

In general, the LbL scattering amplitude is a complicated object for calculations. It is a sum of different diagrams, the quark loop, the meson exchanges, the meson loops and the iterations of these processes. Fortunately, already in the first papers [4,5,6, devoted to the calculation of the hadronic LbL contribution, it was recognized that these numerous terms show a hierarchy. This is related to existence of two small parameters: the inverse number of colors $1 / N_{c}$ and the ratio of the characteristic internal momentum to the chiral symmetry parameter $m_{\mu} /\left(4 \pi f_{\pi}\right) \sim 0.1$. The latter suppresses the multiloop contributions, so that the leading contributions are due to the quark loop diagram and the two-loop diagrams with mesons in the intermediate state. Here the contribution of the diagram with intermediate pion is enhanced by small pion mass in the meson propagator.

Different approaches to the calculation of the pseudoscalar meson contributions to the muon AMM from the light-by-light scattering process are used. These approaches can be separated in two groups. The first one consists of various versions of the vector meson dominance model (VMD) [6,7,8,9,10, and the second one of QCD inspired effective models (EM). They include different versions of the Nambu-Jona-Lasinio model [11,12, the models based on nonperturbative quark-gluon dynamics, like the instanton liquid model [13], the Schwinger-Dyson model [14,15 (DSE) or the holographic models (HM) [16]. To reduce the model dependence of various approaches, different constraints on their parameter space are employed. One kind of important constraints on the models 
follows from the phenomenology of the two-photon widths of the pseudoscalar mesons $\Gamma(P \rightarrow \gamma \gamma)$ and their transition form factors $\mathrm{F}_{P \gamma \gamma^{*}}\left(-M_{P}^{2} ; 0, q^{2}\right)$ first emphasized in 6]. Another set of constraints follows from the large momentum asymptotics for the meson transition form factors [6] and for the total light-by-light scattering amplitude considered in [8, 13], obtained using perturbative QCD. In addition, the model amplitudes have to be consistent with the 4-momentum conservation law. Finally, the model calculations should be tested by calculations of the hadronic vacuum polarization to the muon AMM [17, 18, 19, 20, 21.

The present work is devoted to the calculation of the pseudoscalar mesons contribution of the hadronic lightby-light scattering process to the muon AMM within the nonlocal chiral quark model $(\mathrm{N} \chi \mathrm{QM}), 22,23,24$. In earlier work [13] the contribution of the pion exchange was estimated within this approach in the chiral limit. Now we include the effect of the current quark masses and extend the calculations also to $\eta$ and $\eta^{\prime}$ meson exchanges. As was emphasized in [13], one of the main advantage of the effective model approaches like $\mathrm{N} \chi \mathrm{QM}$ is to control the full kinematical dependence of the LbL amplitude. The paper is organized as follows. In Sect. 2 the basic elements of the effective model is given. In Sect. 3 the properties of the three-point amplitude with arbitrary pseudoscalar meson and photon virtualities are considered. The numerical results on the light-by-light contribution in the pseudoscalar channel obtained within the nonlocal chiral quark model are given in Sect. 4. Sect. 5 is devoted to discussion of the constraints for the LbL amplitude that allow to diminish the model dependence of the estimates. Sect. 6 and 7 contain our results in comparison with other model calculations and conclusions.

\section{$2 \mathbf{N} \chi \mathbf{Q M}$ Lagrangian, $\mathbf{T}$ matrix and $\eta-\eta^{\prime}$ mixing}

The Lagrangian of the $S U(3) \times S U(3)$ chiral quark model has the form 1

$$
\begin{aligned}
\mathcal{L} & =\bar{q}(x)\left(i \hat{\partial}-m_{c}\right) q(x)+\frac{G}{2}\left[J_{S}^{a}(x) J_{S}^{a}(x)+J_{P}^{a}(x) J_{P}^{a}(x)\right] \\
& -\frac{H}{4} T_{a b c}\left[J_{S}^{a}(x) J_{S}^{b}(x) J_{S}^{c}(x)-3 J_{S}^{a}(x) J_{P}^{b}(x) J_{P}^{c}(x)\right], \quad(1)
\end{aligned}
$$

where $q(x)$ are the quark fields, $m_{c}$ is the diagonal matrix of the quark current masses, $G$ and $H$ are the fourand six-quark coupling constants. Second line in the Lagrangian represents the Kobayashi-Maskawa-t'Hooft determinant vertex with the structural constant

$$
T_{a b c}=\frac{1}{6} \epsilon_{i j k} \epsilon_{m n l}\left(\lambda_{a}\right)_{i m}\left(\lambda_{b}\right)_{j n}\left(\lambda_{c}\right)_{k l}
$$

\footnotetext{
${ }^{1}$ For simplicity in this work we do not consider an extended model that includes other structures besides the pseudoscalar (P) and scalar (S) ones.

${ }^{2}$ We consider the isospin limit $m_{c, u}=m_{c, d} \neq m_{c, s}$.
}

where $\lambda_{a}$ are the Gell-Mann matrices for $a=1, . ., 8$ and $\lambda_{0}=\sqrt{2 / 3} I$. The nonlocal structure of the model is introduced via the nonlocal quark currents

$$
J_{M}^{a}(x)=\int d^{4} x_{1} d^{4} x_{2} f\left(x_{1}\right) f\left(x_{2}\right) \bar{q}\left(x-x_{1}\right) \Gamma_{M}^{a} q\left(x+x_{2}\right),
$$

where $M=S, P$ and $\Gamma_{S}^{a}=\lambda^{a}, \Gamma_{P}=i \gamma^{5} \lambda^{a}$, and $f(x)$ is a form factor reflecting the nonlocal properties of the QCD vacuum.

The model (1) can be bosonized using the stationary phase approximation which leads to the system of gap equations for the dynamical quark masses $m_{d, i} \quad(i=$ $u, d, s)$

$$
\begin{aligned}
m_{d, u}+G S_{u}+\frac{H}{2} S_{u} S_{s} & =0 \\
m_{d, s}+G S_{s}+\frac{H}{2} S_{u}^{2} & =0
\end{aligned}
$$

with

$$
S_{i}=-8 N_{c} \int \frac{d_{E}^{4} k}{(2 \pi)^{4}} \frac{f^{2}\left(k^{2}\right) m_{i}\left(k^{2}\right)}{D_{i}\left(k^{2}\right)}
$$

where $m_{i}\left(k^{2}\right)=m_{c, i}+m_{d, i} f^{2}\left(k^{2}\right), D_{i}\left(k^{2}\right)=k^{2}+m_{i}^{2}\left(k^{2}\right)$ is the dynamical quark propagator obtained by solving the Schwinger-Dyson equation, $f\left(k^{2}\right)$ is the nonlocal form factor in the momentum representation.

The vertex functions and the meson masses can be found from the Bethe-Salpeter equation. For the separable interaction (11) the quark-antiquark scattering matrix in pseudoscalar channel becomes

$$
\begin{aligned}
& \mathbf{T}=\hat{\mathbf{T}}\left(p^{2}\right) \delta^{4}\left(p_{1}+p_{2}-\left(p_{3}+p_{4}\right)\right) \prod_{i=1}^{4} f\left(p_{i}^{2}\right), \\
& \hat{\mathbf{T}}\left(p^{2}\right)=i \gamma_{5} \lambda_{k}\left(\frac{1}{-\mathbf{G}^{-1}+\mathbf{\Pi}\left(p^{2}\right)}\right)_{k l} i \gamma_{5} \lambda_{l},
\end{aligned}
$$

where $p_{i}$ are the momenta of external quark lines, $\mathbf{G}$ and $\boldsymbol{\Pi}\left(p^{2}\right)$ are the corresponding matrices of the four-quark coupling constants and the polarization operators of pseudoscalar mesons $\left(p=p_{1}+p_{2}=p_{3}+p_{4}\right)$. The meson masses can be found from the zeros of determinant $\operatorname{det}\left(\mathbf{G}^{-1}-\right.$ $\left.\boldsymbol{\Pi}\left(-M^{2}\right)\right)=0$ and the $\hat{\mathbf{T}}$-matrix for the system of pseudoscalar mesons can be expressed in the form

$$
\hat{\mathbf{T}}\left(p^{2}\right)=\sum_{a=\pi^{0}, \eta, \eta^{\prime}} \frac{\bar{V}_{a}\left(p^{2}\right) \otimes V_{a}\left(p^{2}\right)}{-\left(p^{2}+M_{a}^{2}\right)}
$$

where $M_{a}$ are the meson masses, $V_{a}\left(p^{2}\right)$ are the vertex functions $\left(\bar{V}_{a}\left(p^{2}\right)=\gamma^{0} V_{a}^{\dagger}\left(p^{2}\right) \gamma^{0}\right)$. In general case of three unequal quark masses it is necessary to solve the $\pi^{0}-\eta-\eta^{\prime}$ (or $\eta^{0}-\eta^{3}-\eta^{8}$ ) system factorizing in the isospin limit into the $\pi^{0}$ and $\eta-\eta^{\prime}$ systems. For the $\eta-\eta^{\prime}$ system it is convenient to diagonalize the scattering matrix by orthogonal transformation

$$
\left(\begin{array}{c}
\eta \\
\eta^{\prime}
\end{array}\right)=\left(\begin{array}{cc}
\cos \theta & -\sin \theta \\
\sin \theta & \cos \theta
\end{array}\right)\left(\begin{array}{l}
\eta_{8} \\
\eta_{0}
\end{array}\right)
$$


As a result, the mesonic vertex functions introduced in (5) become

$$
\begin{aligned}
V_{\pi^{0}}\left(p^{2}\right) & =i \gamma_{5} g_{\pi}\left(p^{2}\right) \lambda_{3}, \\
V_{\eta}\left(p^{2}\right) & =i \gamma_{5} g_{\eta}\left(p^{2}\right)\left(\lambda_{8} \cos \theta\left(p^{2}\right)-\lambda_{0} \sin \theta\left(p^{2}\right)\right), \\
V_{\eta^{\prime}}\left(p^{2}\right) & =i \gamma_{5} g_{\eta^{\prime}}\left(p^{2}\right)\left(\lambda_{8} \sin \theta\left(p^{2}\right)+\lambda_{0} \cos \theta\left(p^{2}\right)\right),
\end{aligned}
$$

where $g_{a}\left(p^{2}\right)$ and $\theta\left(p^{2}\right)$ are the meson renormalization constants and mixing angles depending on the meson virtuality. The renormalization constants are defined through the unrenormalized meson propagators $D_{a}\left(p^{2}\right)$ as

$$
g_{a}^{2}\left(p^{2}\right)=-\left(p^{2}+M_{a}^{2}\right) D_{a}\left(p^{2}\right)
$$

The G-matrix elements have the form

$$
\begin{aligned}
G_{00} & =G-\frac{H}{3}\left(2 S_{u}+S_{s}\right), \\
G_{88} & =G+\frac{H}{6}\left(4 S_{u}-S_{s}\right), \\
G_{08} & =G_{80}=\frac{\sqrt{2}}{6} H\left(S_{u}-S_{s}\right), \\
G_{\pi} & =G_{33}=G+\frac{H}{2} S_{s} .
\end{aligned}
$$

The matrix $\boldsymbol{\Pi}\left(p^{2}\right)$ is diagonal in the quark-flavor basis and in the singlet-triplet-octet basis it is given by

$$
\begin{aligned}
& \Pi_{00}\left(p^{2}\right)=\frac{1}{3}\left(2 \Pi_{u u}\left(p^{2}\right)+\Pi_{s s}\left(p^{2}\right)\right), \\
& \Pi_{88}\left(p^{2}\right)=\frac{1}{3}\left(\Pi_{u u}\left(p^{2}\right)+2 \Pi_{s s}\left(p^{2}\right)\right), \\
& \Pi_{08}\left(p^{2}\right)=\Pi_{80}\left(p^{2}\right)=\frac{\sqrt{2}}{3}\left(\Pi_{u u}\left(p^{2}\right)-\Pi_{s s}\left(p^{2}\right)\right), \\
& \Pi_{\pi}\left(p^{2}\right)=\Pi_{33}\left(p^{2}\right)=\Pi_{u u}\left(p^{2}\right),
\end{aligned}
$$

where the polarization operators are

$$
\begin{aligned}
\Pi_{i j}\left(p^{2}\right) & =8 N_{c} \int \frac{d_{E}^{4} k}{(2 \pi)^{4}} \frac{f^{2}\left(k_{+}^{2}\right) f^{2}\left(k_{-}^{2}\right)}{D_{i}\left(k_{+}^{2}\right) D_{j}\left(k_{-}^{2}\right)} \times \\
& \times\left[\left(k_{+} \cdot k_{-}\right)+m_{i}\left(k_{+}^{2}\right) m_{j}\left(k_{-}^{2}\right)\right],
\end{aligned}
$$

and $k_{ \pm}=k \pm p / 2$. The unrenormalized mesonic propagators for the pseudoscalar mesons are

$$
\begin{aligned}
& D_{\pi}^{-1}\left(p^{2}\right)=-G_{\pi}^{-1}+\Pi_{\pi}\left(p^{2}\right) \\
& D_{\eta, \eta^{\prime}}^{-1}\left(p^{2}\right)=\frac{1}{2}\left[(A+C) \mp \sqrt{(A-C)^{2}+4 B^{2}}\right], \\
& A=-G_{88} / \operatorname{det}(\mathbf{G})+\Pi_{00}\left(p^{2}\right), \\
& B=+G_{08} / \operatorname{det}(\mathbf{G})+\Pi_{08}\left(p^{2}\right), \\
& C=-G_{00} / \operatorname{det}(\mathbf{G})+\Pi_{88}\left(p^{2}\right) \\
& \operatorname{det}(\mathbf{G})=G_{00} G_{88}-G_{08}^{2} .
\end{aligned}
$$

The meson mixing angle depends on the virtuality

$$
\theta\left(p^{2}\right)=\frac{1}{2} \arctan \left[\frac{2 B}{A-C}\right]-\frac{\pi}{2} \Theta(A-C) .
$$

Therefore $\theta_{\eta}=\theta\left(-M_{\eta}^{2}\right)$ and $\theta_{\eta^{\prime}}=\theta\left(-M_{\eta^{\prime}}^{2}\right)$ are different for the on-shell $\eta$ and $\eta^{\prime}$ mesons.

For numerical estimations we use the Gaussian nonlocal form factor

$$
f\left(k^{2}\right)=\exp \left(-k^{2} / 2 \Lambda^{2}\right)
$$

and the model parameters obtained in 24. In that work these parameters (the current quark masses $m_{c, i}$, the coupling constants $G$ and $H$, and the nonlocality scale $\Lambda$ ) are fixed by requiring that the model reproduces correctly the measured values of the pion and kaon masses, the pion decay constant $f_{\pi}$, and the $\eta^{\prime}$ mass (parameter sets $\mathrm{G}_{I}, \mathrm{G}_{I V}$ ) or the $\eta^{\prime} \rightarrow \gamma \gamma$ decay constant $g_{\eta^{\prime} \gamma \gamma}\left(\right.$ sets $\left.\mathrm{G}_{I I}, \mathrm{G}_{I I I}\right)$. For completeness, we present the parameter sets $G_{I-I V}$ and the basic meson properties in Tables 1 and 2 as they were determined in [24]. The sets $\mathrm{G}_{I}, \mathrm{G}_{I V}$ vary by different input for the nonstrange current quark mass, while $\mathrm{G}_{I I}$, $\mathrm{G}_{I I I}$ are two solutions of the same fitting procedure.

\section{Anomalous triangles}

By using the nonlocal chiral quark mode 3 , the triangular diagram with external pseudoscalar meson and two photon legs with arbitrary virtualities (Fig. 1) can be written as

$$
\begin{aligned}
& A\left(\gamma^{*}\left(q_{1}, \epsilon_{1}\right) \gamma^{*}\left(q_{2}, \epsilon_{2}\right) \rightarrow P^{*}(p)\right)= \\
& \quad-i e^{2} \varepsilon_{\mu \nu \rho \sigma} \epsilon_{1}^{\mu} \epsilon_{2}^{\nu} q_{1}^{\rho} q_{2}^{\sigma} \mathrm{F}_{P^{*} \gamma^{*} \gamma^{*}}\left(p^{2} ; q_{1}^{2}, q_{2}^{2}\right),
\end{aligned}
$$

with the photon momenta $q_{1,2}$ and the polarization vectors $\epsilon_{1,2}, p=q_{1}+q_{2}$. For different pseudoscalar meson states one has

$$
\begin{aligned}
& \mathrm{F}_{\pi_{0}^{*} \gamma^{*} \gamma^{*}}\left(p^{2} ; q_{1}^{2}, q_{2}^{2}\right)=g_{\pi}\left(p^{2}\right) F_{u}\left(p^{2} ; q_{1}^{2}, q_{2}^{2}\right) \\
& \mathrm{F}_{\eta^{*} \gamma^{*} \gamma^{*}}\left(p^{2} ; q_{1}^{2}, q_{2}^{2}\right)=\frac{g_{\eta}\left(p^{2}\right)}{3 \sqrt{3}} \times \\
& \quad \times\left[\left(5 F_{u}\left(p^{2} ; q_{1}^{2}, q_{2}^{2}\right)-2 F_{s}\left(p^{2} ; q_{1}^{2}, q_{2}^{2}\right)\right) \cos \theta\left(p^{2}\right)-\right. \\
& \left.\quad-\sqrt{2}\left(5 F_{u}\left(p^{2} ; q_{1}^{2}, q_{2}^{2}\right)+F_{s}\left(p^{2} ; q_{1}^{2}, q_{2}^{2}\right)\right) \sin \theta\left(p^{2}\right)\right] \\
& \mathrm{F}_{\eta^{\prime *} \gamma^{*} \gamma^{*}}\left(p^{2} ; q_{1}^{2}, q_{2}^{2}\right)=\frac{g_{\eta^{\prime}}\left(p^{2}\right)}{3 \sqrt{3}} \times \\
& \quad \times\left[\left(5 F_{u}\left(p^{2} ; q_{1}^{2}, q_{2}^{2}\right)-2 F_{s}\left(p^{2} ; q_{1}^{2}, q_{2}^{2}\right)\right) \sin \theta\left(p^{2}\right)+\right. \\
& \left.\quad+\sqrt{2}\left(5 F_{u}\left(p^{2} ; q_{1}^{2}, q_{2}^{2}\right)+F_{s}\left(p^{2} ; q_{1}^{2}, q_{2}^{2}\right)\right) \cos \theta\left(p^{2}\right)\right]
\end{aligned}
$$

with

\footnotetext{
${ }^{3}$ For the pion, the corresponding vertex in the chiral limit has been considered in 25 .
} 
A.E. Dorokhov et al.: The pseudoscalar hadronic channel contribution of the light-by-light process ...

\begin{tabular}{|c|c|c|c|c|c|c|c|}
\hline set & $\begin{array}{c}m_{c, u} \\
{[\mathrm{MeV}]}\end{array}$ & $\begin{array}{c}m_{d, u} \\
{[\mathrm{MeV}]}\end{array}$ & $\begin{array}{c}m_{c, s} \\
{[\mathrm{MeV}]}\end{array}$ & $\begin{array}{c}m_{d, s} \\
{[\mathrm{MeV}]}\end{array}$ & $\begin{array}{c}\Lambda \\
{[\mathrm{MeV}]}\end{array}$ & $G \Lambda^{2}$ & $H \Lambda^{5}$ \\
\hline $\mathrm{G}_{I}$ & 8.5 & 304.5 & 223 & 427 & 709 & 21.986 & -1670.19 \\
$\mathrm{G}_{I I}$ & 8.5 & 304.5 & 223 & 439 & 709 & 22.898 & -1557.28 \\
$\mathrm{G}_{I I I}$ & 8.5 & 304.5 & 223 & 422 & 709 & 21.605 & -1717.59 \\
$\mathrm{G}_{I V}$ & 7.5 & 287.5 & 199 & 408 & 768 & 20.896 & -1721.69 \\
\hline
\end{tabular}

Table 1. The model parameter sets obtained in [24].

\begin{tabular}{|c|c|c|c|c|c|c|}
\hline set & $\begin{array}{c}M_{\pi} \\
{[\mathrm{MeV}]}\end{array}$ & $\begin{array}{c}M_{\eta} \\
{[\mathrm{MeV}]}\end{array}$ & $\begin{array}{c}M_{\eta^{\prime}} \\
{[\mathrm{MeV}]}\end{array}$ & $\begin{array}{c}g_{\pi \gamma \gamma} \\
{\left[\mathrm{GeV}^{-1}\right]}\end{array}$ & $\begin{array}{c}g_{\eta \gamma \gamma} \\
{\left[\mathrm{GeV}^{-1}\right]}\end{array}$ & $\begin{array}{c}g_{\eta^{\prime} \gamma \gamma} \\
{\left[\mathrm{GeV}^{-1}\right]}\end{array}$ \\
\hline $\mathrm{G}_{I}$ & 138.9 & 516.5 & 958.4 & 0.2706 & 0.3082 & 0.3752 \\
$\mathrm{G}_{I I}$ & 138.9 & 505.4 & 878.6 & 0.2706 & 0.3259 & 0.3401 \\
$\mathrm{G}_{I I I}$ & 138.9 & 520.7 & 1006.4 & 0.2706 & 0.3011 & 0.3489 \\
$\mathrm{G}_{I V}$ & 139.0 & 522.1 & $>739.7$ & 0.2713 & 0.3068 & \\
\hline $\exp$ & 134.9766 & 547.8533 & 957.78 & 0.2744 & 0.2726 & 0.3423 \\
& \pm 0.0006 & \pm 0.024 & \pm 0.06 & ${ }_{-0.008}^{+0.009}$ & \pm 0.008 & \pm 0.014 \\
\hline
\end{tabular}

Table 2. The basic meson properties for different parametrizations obtained in 24].

$$
\begin{gathered}
F_{i}\left(p^{2} ; q_{1}^{2}, q_{2}^{2}\right)=8 \int \frac{d_{E}^{4} k}{(2 \pi)^{4}} \frac{f\left(k_{1}^{2}\right) f\left(k_{2}^{2}\right)}{D_{i}\left(k_{1}^{2}\right) D_{i}\left(k_{2}^{2}\right) D_{i}\left(k^{2}\right)} \times \\
\times\left[m_{i}\left(k^{2}\right)-\mathrm{m}_{i}^{(1)}\left(k_{1}, k\right) J_{1}-\mathrm{m}_{i}^{(1)}\left(k_{2}, k\right) J_{2}\right] \\
J_{1}=k^{2}+\frac{q_{2}^{2}\left(k q_{1}\right)\left(k_{1} q_{1}\right)-q_{1}^{2}\left(k q_{2}\right)\left(k_{1} q_{2}\right)}{q_{1}^{2} q_{2}^{2}-\left(q_{1} q_{2}\right)^{2}} \\
J_{2}=k^{2}+\frac{q_{1}^{2}\left(k q_{2}\right)\left(k_{2} q_{2}\right)-q_{2}^{2}\left(k q_{1}\right)\left(k_{2} q_{1}\right)}{q_{1}^{2} q_{2}^{2}-\left(q_{1} q_{2}\right)^{2}}
\end{gathered}
$$

where $k_{1}=k+q_{1}, k_{2}=k-q_{2}, \mathrm{~m}_{i}^{(1)}(k, p)=\left(m_{i}\left(k^{2}\right)-\right.$ $\left.m_{i}\left(p^{2}\right)\right) /\left(k^{2}-p^{2}\right)$ is the first order finite-difference derivative. From (17) one can easily obtain the expressions for some special kinematics

$$
\begin{gathered}
F_{i}\left(q_{1}^{2} ; q_{1}^{2}, 0\right)=8 \int \frac{d_{E}^{4} k}{(2 \pi)^{4}} \frac{f\left(k_{1}^{2}\right) f\left(k^{2}\right)}{D_{i}\left(k_{1}^{2}\right) D_{i}^{2}\left(k^{2}\right)} \times \\
\times\left[m_{i}\left(k^{2}\right)-\mathrm{m}_{i}^{(1)}\left(k_{1}, k\right) \bar{J}_{1}-m_{i}^{\prime}\left(k^{2}\right) \bar{J}_{2}\right], \\
\bar{J}_{1}\left(k, q_{1}\right)=\left(k q_{1}\right)+\frac{2}{3}\left[k^{2}+2 \frac{\left(k q_{1}\right)^{2}}{q_{1}^{2}}\right], \\
\bar{J}_{2}=\frac{4}{3}\left[k^{2}-\frac{\left(k q_{1}\right)^{2}}{q_{1}^{2}}\right], \\
F_{i}(0 ; 0,0)=\frac{1}{m_{d, i}}\left[\frac{1}{4 \pi^{2}}-\right. \\
\left.-8 m_{c, i} \int \frac{d_{E}^{4} k}{(2 \pi)^{4}} \frac{m_{i}\left(k^{2}\right)-2 m_{i}^{\prime}\left(k^{2}\right) k^{2}}{D_{i}^{3}\left(k^{2}\right)}\right],
\end{gathered}
$$

In particular, the kinematics displayed in (18) is of special interest for the hadronic exchange LbL calculations (see Eq. (20) below). In (19) the first term is due to the axial anomaly, while the second term represents the correction due to explicit breaking of the chiral symmetry by current quark mass.

\section{Light-by-light hadronic contribution to the muon AMM in the pseudoscalar meson channel}

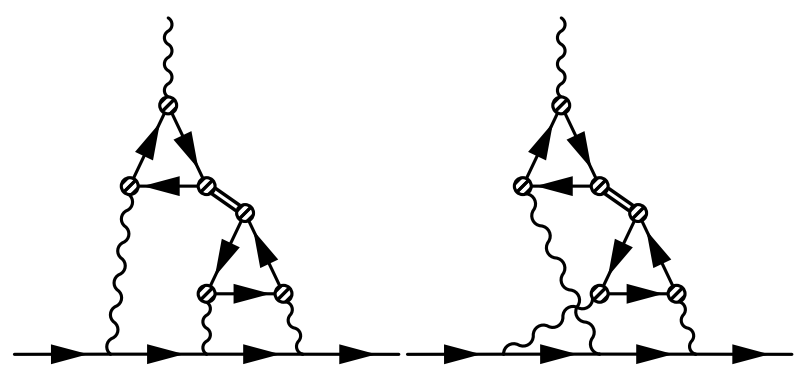

Fig. 1. Light-by-light contribution from intermediate pseudoscalar meson exchanges.

The light-by-light contribution due to exchanges of the light hadrons in the intermediate pseudoscalar channel to the muon AMM is shown in Fig. 1 and can be written in the form 26$]$

$$
\begin{aligned}
& a_{\mu}^{\mathrm{LbL}, \mathrm{PS}}=-\frac{2 \alpha^{3}}{3 \pi^{2}} \int_{0}^{\infty} d q_{1}^{2} \int_{0}^{\infty} d q_{2}^{2} \int_{-1}^{1} d t \sqrt{1-t^{2}} \frac{1}{q_{3}^{2}} \times \\
& \times \sum_{a=\pi^{0}, \eta, \eta^{\prime}}\left[2 \frac{\mathrm{F}_{a^{*} \gamma^{*} \gamma^{*}}\left(q_{2}^{2} ; q_{1}^{2}, q_{3}^{2}\right) \mathrm{F}_{a^{*} \gamma^{*} \gamma}\left(q_{2}^{2} ; q_{2}^{2}, 0\right)}{q_{2}^{2}+M_{a}^{2}} I_{1}\right. \\
& \left.+\frac{\mathrm{F}_{a^{*} \gamma^{*} \gamma^{*}}\left(q_{3}^{2} ; q_{1}^{2}, q_{2}^{2}\right) \mathrm{F}_{a^{*} \gamma^{*} \gamma}\left(q_{3}^{2} ; q_{3}^{2}, 0\right)}{q_{3}^{2}+M_{a}^{2}} I_{2}\right]
\end{aligned}
$$

where $q_{3}=-\left(q_{1}+q_{2}\right)$. The functions $I_{1}$ and $I_{2}$ are 


$$
\begin{aligned}
I_{1} & =q_{1}^{2}\left[\left\langle\frac{1}{D_{1}}\right\rangle\left(\frac{\left(q_{1} q_{2}\right)}{2}-q_{2}^{2}\left(1-t^{2}\right)\right)+\left\langle\frac{p q_{2}}{D_{1}}\right\rangle+\right. \\
+ & \left.\left\langle\frac{1}{D_{1} \cdot D_{2}}\right\rangle q_{2}^{2}\left(1-t^{2}\right)\left(q_{2}^{2}-2 M_{\mu}^{2}\right)\right]-\frac{\left(q_{1} q_{2}\right)}{2} \\
& I_{2}=2 q_{2}^{2}\left[\left\langle\frac{1}{D_{2}}\right\rangle\left(q_{1}^{2}+\left(q_{1} q_{2}\right)\right)-\left\langle\frac{p q_{1}}{D_{2}}\right\rangle-\right. \\
& \left.-\left\langle\frac{1}{D_{1} \cdot D_{2}}\right\rangle q_{1}^{2}\left(q_{1}^{2}+\left(q_{1} q_{2}\right)+M_{\mu}^{2}\left(1-t^{2}\right)\right)\right]+ \\
& +\left\langle\frac{1}{D_{1}}\right\rangle q_{1}^{2}\left(q_{1} q_{2}\right)-\left(q_{1} q_{2}\right)
\end{aligned}
$$

are obtained [26] after averaging over the directions of muon momentum $p$

$$
\langle\ldots\rangle=\frac{1}{2 \pi^{2}} \int d \Omega(\widehat{p}) \ldots
$$

In (21) and (22) the notations are $\left(D_{1}=\left(p+q_{1}\right)^{2}+M_{\mu}^{2}\right.$, $\left.D_{2}=\left(p-q_{2}\right)^{2}+M_{\mu}^{2}\right)$

$$
\begin{aligned}
& \left\langle\frac{1}{D_{1}}\right\rangle=\frac{R_{1}-1}{2 M_{\mu}^{2}}, \quad\left\langle\frac{1}{D_{2}}\right\rangle=\frac{R_{2}-1}{2 M_{\mu}^{2}} \\
& \left\langle\frac{1}{D_{1} \cdot D_{2}}\right\rangle=\frac{1}{M_{\mu}^{2}\left|q_{1}\right|\left|q_{2}\right| x} \arctan \left[\frac{z x}{1-z t}\right] \\
& \left\langle\frac{p q_{1}}{D_{2}}\right\rangle=-\left(q_{1} q_{2}\right) \frac{\left(1-R_{2}\right)^{2}}{8 M_{\mu}^{2}}, \\
& \left\langle\frac{p q_{2}}{D_{1}}\right\rangle=\left(q_{1} q_{2}\right) \frac{\left(1-R_{1}\right)^{2}}{8 M_{\mu}^{2}}, \\
& t=\frac{\left(q_{1} q_{2}\right)}{\left|q_{1}\right|\left|q_{2}\right|}, \quad x=\sqrt{1-t^{2}}, R_{i}=\sqrt{1+\frac{4 M_{\mu}^{2}}{q_{i}^{2}}} \\
& z=\frac{q_{1} q_{2}}{4 M_{\mu}^{2}}\left(1-R_{1}\right)\left(1-R_{2}\right),
\end{aligned}
$$

and $M_{\mu}$ is the muon mass $\left(p^{2}=-M_{\mu}^{2}\right)$.

It is instructive to investigate the pion contribution to the muon AMM $a_{\mu}^{\mathrm{LbL}, \pi^{0}}$ for the $S U(2)$ reduction of the nonlocal model (10). In this case, in the isospin limit, there are three model parameters: the current $m_{c, u}$ and dynamical $m_{d, u}$ quark masses and the nonlocality parameter $\Lambda$. Varying one parameter, say $m_{d, u}$, in some region, one can fix other parameters by using as input the pion mass and the two-photon decay constant of the neutral pion 4 . The pion mass is known with high accuracy, but

\footnotetext{
${ }^{4}$ The properties of the charged pion, namely the mass and the weak decay constant, are used as a rule for fixing the model parameters. In general, the difference between fixing the parameter space for the charged pion or for the neutral pion is
}

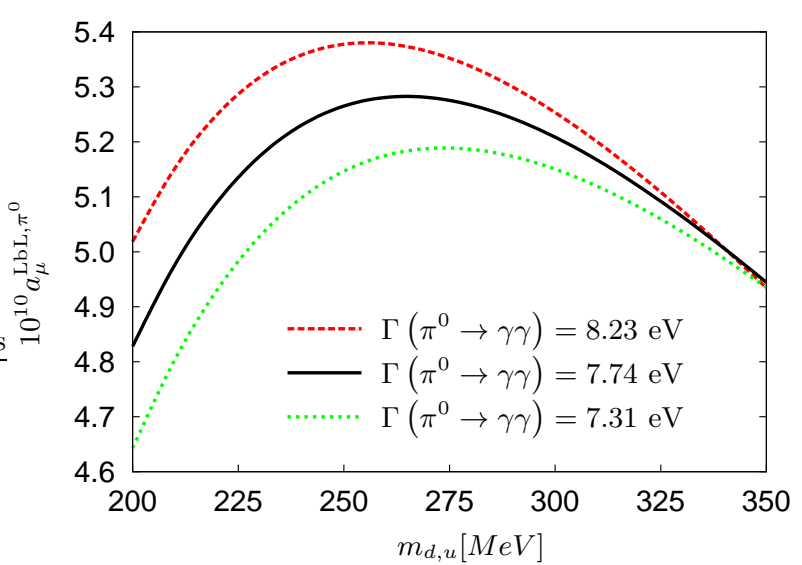

Fig. 2. Light-by-light contribution to the muon AMM from the neutral pion exchange as a function of the dynamical quark mass. The band between dotted lines corresponds to the error interval for the pion two-photon width.

the two-photon decay constant has an experimental uncertainty at the level of $3 \%$. We investigate the dependence of $a_{\mu}^{\mathrm{LbL}, \pi^{0}}$ on this uncertainty. Namely, the dynamical quark mass is taken in the typical interval 200-350 $\mathrm{MeV}$ and then other parameters are fitted by the pion mass and the two-photon decay constant in correspondence with the pion lifetime given within the error range in [27]. The dependency of $a_{\mu}^{\mathrm{LbL}, \pi^{0}}$ as a function of $m_{d, u}$ within the error interval for the two-photon decay constant is shown in Fig. 2 and, thus, we get our conservative estimate $a_{\mu}^{\mathrm{LbL}, \pi^{0}}=(5.01 \pm 0.37) \cdot 10^{-10}$.

The results for the nonlocal $S U(3)$ model (1) with the parameter sets $\mathrm{G}_{I-I V}$ are given in Table 3 . Note, that the results for $a_{\mu}^{\mathrm{LbL}, \pi^{0}}$ are in the error range consistent with the results obtained in the nonlocal $S U(2)$ model and that $a_{\mu}^{\mathrm{LbL}, \eta}$ and $a_{\mu}^{\mathrm{LbL}, \eta^{\prime}}$ are one order less than the contribution to the muon AMM due to the pion.

\begin{tabular}{|c|c|c|c|c|c|}
\hline set & $\pi^{0}$ & $\eta$ & $\eta^{\prime}$ & $\eta+\eta^{\prime}$ & $\pi^{0}+\eta+\eta^{\prime}$ \\
\hline $\mathrm{G}_{I}$ & 5.05 & 0.55 & 0.27 & 0.82 & 5.87 \\
$\mathrm{G}_{I I}$ & 5.05 & 0.59 & 0.48 & 1.08 & 6.13 \\
$\mathrm{G}_{I I I}$ & 5.05 & 0.53 & 0.18 & 0.71 & 5.76 \\
$\mathrm{G}_{I V}$ & 5.10 & 0.49 & 0.25 & 0.74 & 5.84 \\
\hline
\end{tabular}

Table 3. The contribution of pseudoscalar mesons to the muon $\mathrm{AMM} a_{\mu}^{\mathrm{LbL}}$ obtained within $\mathrm{N} \chi \mathrm{QM}$ for different parametrizations. All numbers are given in $10^{-10}$.

due to small isotopic invariance breaking corrections induced by the electro-magnetic interaction and inequality of the nonstrange quark masses. We believe that for the problem under consideration it is better to fix the model parameters by the neutral pion properties. 


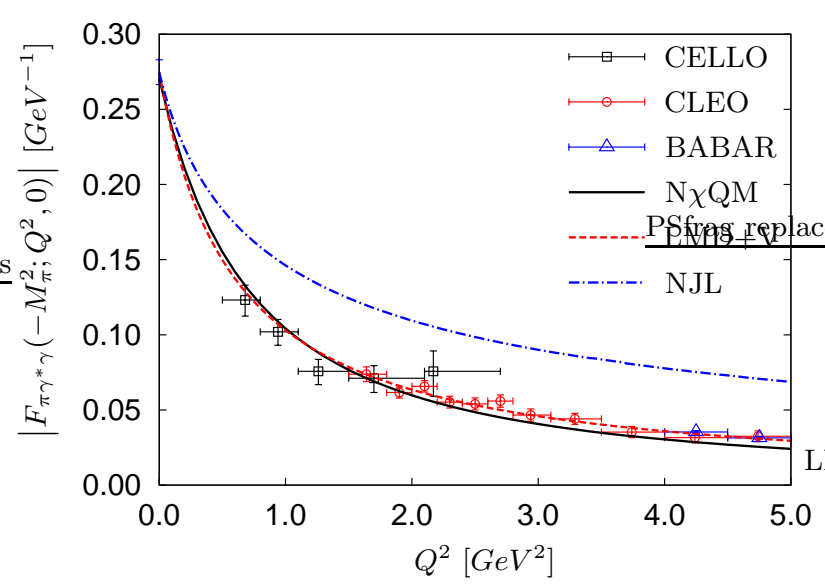

Fig. 3. The $\gamma^{*} \gamma \rightarrow \pi^{0}$ transition form factor in $\mathrm{N} \chi \mathrm{QM}$ (this work, parameter set $\mathrm{G}_{I}$ is used), LMD+V 7] and NJL 12 models in comparison with the CELLO 28, CLEO 29 and BABAR [30] data.

\section{Constraints on $P \gamma \gamma$ vertex and LbL amplitude}

A first phenomenological constraint on the anomalous vertex $\mathrm{F}_{P^{*} \gamma^{*} \gamma^{*}}\left(q_{3}^{2} ; q_{1}^{2}, q_{2}^{2}\right)$ is put when both photons and the meson are on-shell. The vertex $\mathrm{F}_{P \gamma \gamma}\left(-M_{P}^{2} ; 0,0\right)$ is normalized by the experimentally measured two-photon decay widths

$$
\mathrm{F}_{P \gamma \gamma}\left(-M_{P}^{2} ; 0,0\right) \equiv g_{P \gamma \gamma}=\sqrt{\frac{64 \pi \Gamma(P \rightarrow \gamma \gamma)}{(4 \pi \alpha)^{2} M_{P}^{3}}}
$$

A second phenomenological constraint is, that the vertex for special kinematics, when the meson and one of the photon are on-shell, $\mathrm{F}_{P \gamma \gamma^{*}}\left(-M_{P}^{2} ; 0, q^{2}\right)$, has to fit the data on the pseudoscalar meson transition form factors available from the measurements of the CELLO [28], CLEO [29] and BABAR 30,31] collaborations. The transition form-factors for $\pi, \eta, \eta^{\prime}$ mesons evaluated in the $\mathrm{N} \chi \mathrm{QM}$ for the $\mathrm{G}_{I}$ parameter set are shown in Figs. 3415. One can see the reasonable agreement of the $\mathrm{N} \chi \mathrm{QM}$ (this work) and $\mathrm{LMD}+\mathrm{V}(\mathrm{VMD}) 6$ [7] models predictions with existing experimental data, while the NJL model with the parameter set taken in [12] is not perfect in explanation of the data.

A first QCD constraint 35] is, that at large photon virtualities $q_{1}^{2}+q_{2}^{2} \rightarrow \infty$ the meson transition form factor has the asymptotic behavior as

$$
\mathrm{F}_{P \gamma^{*} \gamma^{*}}\left(-M_{P}^{2} ; q_{1}^{2}, q_{2}^{2}\right) \sim 1 /\left(q_{1}^{2}+q_{2}^{2}\right)
$$

\footnotetext{
${ }^{5}$ As was discussed in $32,33,34$, new, very interesting data of the BABAR collaboration [30,31] at high momentum transfer of the photon do not affect very much the low energy observables.

${ }^{6}$ Some details about the $\mathrm{LMD}+\mathrm{V}$ and VMD models are given in Sect. 6.
}

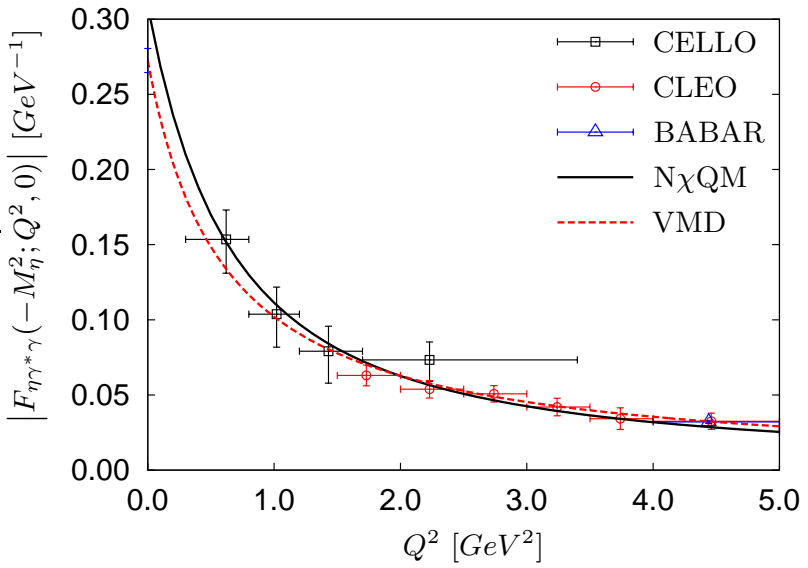

Fig. 4. The $\gamma^{*} \gamma \rightarrow \eta$ transition form factor in $\mathrm{N} \chi \mathrm{QM}$ (this work, $\mathrm{G}_{I}$ set) and VMD [7 models in comparison with the CELLO 28, CLEO 29] and BABAR 31] data.

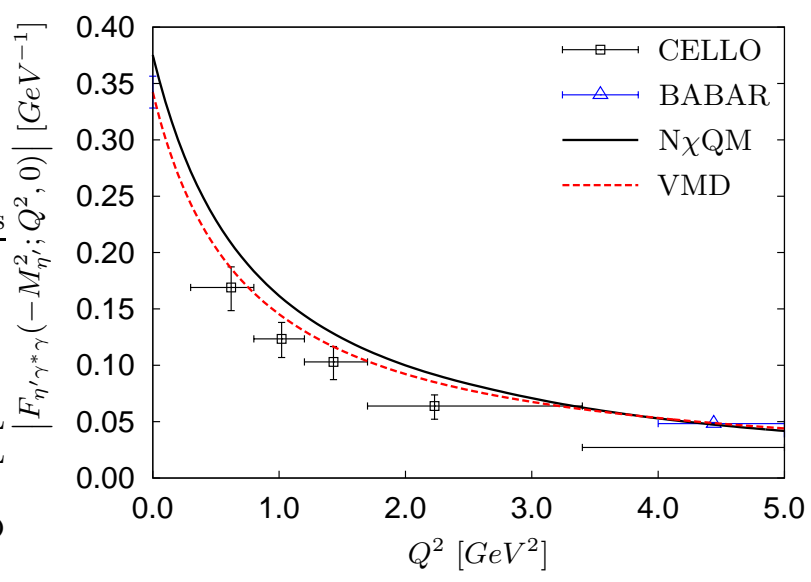

Fig. 5. The $\gamma^{*} \gamma \rightarrow \eta^{\prime}$ transition form factor in $\mathrm{N} \chi \mathrm{QM}$ (this work, $\mathrm{G}_{I}$ set) and VMD [7] models in comparison with the CELLO 28 and BABAR 31] data points.

and for symmetric kinematics it fixes the asymptotic coefficient. In the case of pion one has

$$
\begin{aligned}
& \mathrm{F}_{\pi \gamma^{*} \gamma^{*}}(-\left.M_{\pi}^{2} ; q^{2}, q^{2}\right) \approx \frac{1}{q^{2}} \frac{2 f_{\pi}}{3}\left(1-\frac{5}{6} \frac{\alpha_{s}\left(q^{2}\right)}{\pi}+\right. \\
&\left.+O\left(\alpha_{s}^{2}\right)+O\left(q^{-2}\right)\right)
\end{aligned}
$$

Note, that even, if both in the $\mathrm{N} \chi \mathrm{QM}$ and the $\mathrm{LMD}+\mathrm{V}$ approaches the $1 / q^{2}$ behavior is satisfied and power corrections are taken into account, these models are still incomplete to describe the radiative corrections $O\left(\alpha_{s}\right)$ calculated within the perturbative QCD 36.

A second QCD constraint is to match the total lightby-light amplitude with three virtual $\left(q_{i}, i=1,2,3\right)$ and one external magnetic $\left(q_{4} \rightarrow 0\right)$ field

$$
\mathcal{M}\left(q_{1}^{2}, q_{2}^{2}, q_{3}^{2}\right) \equiv \alpha^{2} N_{c} \operatorname{Tr}\left[\widehat{Q}^{4}\right] \mathcal{A}\left(q_{1}^{2}, q_{2}^{2}, q_{3}^{2}\right)
$$

to the perturbative QCD asymptotics for the configurations when some of photon virtualities are large 8 . There 
are only two distinct kinematic regimes in the light-bylight scattering amplitudes: the Euclidean momenta of the three photons are comparable in magnitude $q_{1}^{2} \approx q_{2}^{2} \approx$ $q_{3}^{2} \gg \Lambda_{\mathrm{QCD}}^{2}$, or one of the momenta is much smaller than the other two $q_{1}^{2} \approx q_{2}^{2} \gg q_{3}^{2} \gg \Lambda_{\mathrm{QCD}}^{2}$. The second limit was analyzed in [8] using the OPE with the result that the amplitude is factorized in the short-distance factor $\sim 1 / q_{1}^{2}$ times the nondiagonal correlator $(V A \widetilde{V})$ between the axial and electromagnetic current in the external magnetic field

$$
\begin{aligned}
\mathcal{A}_{P S}\left(q_{1}^{2}, q_{2}^{2}, q_{3}^{2}\right) \stackrel{q_{1}^{2} \approx q_{2}^{2} \gg q_{3}^{2}}{=} & =\sum_{a=3,8,0} W^{(a)} G_{2}^{(a)}\left(q_{3}^{2}, q_{2}^{2}, q_{1}^{2}\right)\left\{f_{2} \bar{f}_{1}\right\}\left\{\bar{f} f_{3}\right\}
\end{aligned}
$$

where

$$
\begin{aligned}
& G_{2}^{(a)}\left(q_{3}^{2}, q_{2}^{2}, q_{1}^{2}\right) \stackrel{q_{1}^{2} \approx q_{2}^{2} \gg q_{3}^{2} \gg \Lambda_{\mathrm{QCD}}^{2}}{=} \frac{2}{q_{1}^{2}} w_{L}^{(a)}\left(q_{3}^{2}\right) \\
& W^{(3)}=\frac{1}{4}, \quad W^{(8)}=\frac{1}{12}, \quad W^{(0)}=\frac{2}{3},
\end{aligned}
$$

$w_{L}^{(a)}\left(q_{3}^{2}\right)$ is the longitudinal part of the $V A \widetilde{V}$ correlator with the corresponding flavor structure of the axial current, $f_{i}^{\mu \nu}=q_{i}^{\mu} \epsilon_{i}^{\nu}-q_{i}^{\nu} \epsilon_{i}^{\mu}$ are the field strength tensors, the braces denote traces of the products of the matrices $f_{i}^{\mu \nu}$. In (26), the amplitude $\mathcal{A}_{P S}$ is the part of the total amplitude that is relevant to hadronic exchange in the pseudoscalar channel. The properties of the $V A \widetilde{V}$ correlator was studied in detail in 37] where it was shown that in the chiral limit of massless current quarks, due to properties of the axial anomaly, in the perturbative QCD one has for all $a$

$$
w_{L}^{(a)}\left(q^{2}\right)=\frac{2}{q^{2}},
$$

moreover, these expressions for $w_{L}^{(3,8)}\left(q^{2}\right)$ are exact QCD results for nonsinglet axial currents valid at any $q^{2}$. This result was also confirmed within the effective instanton liquid model $(\mathrm{N} \chi \mathrm{QM})$ 38. In 39] it was also demonstrated how the anomalous longitudinal part of the correlator $w_{L}^{(0)}\left(q^{2}\right)$ is modified at low momenta $\left(q^{2} \lesssim m_{\eta^{\prime}}^{2}\right)$ due to the presence of the $U_{A}(1)$ anomaly. Thus within $\mathrm{OPE}$ one has, independently of $a$, the QCD asymptotic constraint

$$
G_{2}\left(q_{3}^{2}, q_{2}^{2}, q_{1}^{2}\right) \stackrel{q_{1}^{2} \approx q_{2}^{2} \gg q_{3}^{2} \gg \Lambda_{\mathrm{QCD}}^{2}}{=} \frac{4}{q_{1}^{2} q_{3}^{2}}
$$

The function $G_{2}\left(q_{3}^{2}, q_{2}^{2}, q_{1}^{2}\right)$ was given in [8] as a result of explicit calculations in perturbative theory for the lightby-light scattering amplitude where the photon-photon interaction is mediated by the loop of massless quarks. This function is symmetric under the permutation of the last two arguments and in specific kinematics it is reduced to

$$
\begin{aligned}
& G_{2}(s, s, s)=\frac{8}{3 s^{2}} \\
& G_{2}\left(s_{3}, s, s\right)=\frac{8}{s_{3}\left(s_{3}-4 s\right)^{2}}\left[\left(2 s+s_{3}\right) \ln \frac{s_{3}}{s}+\right. \\
& \left.\quad+4 s-s_{3}+2 s\left(s-s_{3}\right) J\left(s_{3}, s, s\right)\right] \\
& G_{2}\left(s, s, s_{1}\right)=\frac{4}{s s_{1}\left(s_{1}-4 s\right)^{2}}\left[\left(4 s^{2}-s_{1}^{2}\right) \ln \frac{s}{s_{1}}+\right. \\
& \left.\quad+2 s\left(4 s-s_{1}\right)-2 s\left(s_{1}^{2}+2 s^{2}-3 s s_{1}\right) J\left(s, s, s_{1}\right)\right]
\end{aligned}
$$

where $s_{i}=q_{i}^{2}$. The triangle function $J\left(s_{3}, s_{2}, s_{1}\right)$ (see, for example, 40]) is symmetric in all its arguments and for the kinematics considered it is equal to

$$
\begin{gathered}
J\left(s_{3}, s, s\right)= \\
=\left\{\begin{array}{c}
\frac{1}{s} \frac{2}{\sqrt{x(1-x / 4)}} \mathrm{Cl}_{2}\left(\arccos \left(1-\frac{x}{2}\right)\right), \text { if } 4 s>s_{3} \\
\frac{1}{s_{3}} \frac{1}{\lambda}\left[2 \ln ^{2}\left(\frac{1-\lambda}{2}\right)-4 \operatorname{Li}_{2}\left(\frac{1-\lambda}{2}\right)-\ln ^{2}(y)+\frac{\pi^{2}}{3}\right] \\
\text { if } 4 s<s_{3},
\end{array}\right.
\end{gathered}
$$

where $x=\frac{s_{3}}{s}(0 \leq x \leq 4), y=\frac{s}{s_{3}}(0 \leq y \leq 1 / 4), \lambda=$ $\sqrt{1-4 y}, \mathrm{Cl}_{2}(\theta)$ is the Clausen integral function

$$
\mathrm{Cl}_{2}(\theta)=-\int_{0}^{\theta} d t \ln \left|2 \sin \frac{t}{2}\right|
$$

and $\mathrm{Li}_{2}(\eta)$ is the dilogarithm (Spence) function

$$
\operatorname{Li}_{2}(\eta)=-\int_{0}^{1} d t \frac{\ln (1-\eta t)}{t}
$$

Asymptotically, when some photon virtualities are large, one has

$$
\begin{aligned}
G_{2}\left(s_{3}, s, s\right) & \stackrel{s \gg s_{3}}{=} \frac{1}{s s_{3}}\left[4+\frac{s_{3}}{s}\left(\frac{4}{3} \ln \left(\frac{s_{3}}{s}\right)-\frac{2}{9}\right)+\right. \\
\left.+O\left(\left(\frac{s_{3}}{s}\right)^{2}\right)\right], & \\
G_{2}\left(s, s, s_{1}\right) & \stackrel{s}{=} \frac{4}{s^{2}}\left[-\frac{1}{3} \ln \left(\frac{s_{1}}{s}\right)+\frac{5}{9}+O\left(\frac{s_{1}}{s}\right)\right],
\end{aligned}
$$


and

$$
\begin{aligned}
& G_{2}\left(s_{3}, s, s\right) \stackrel{s \ll s_{3}}{=}-\frac{8}{s_{3}^{2}}\left[\ln \left(\frac{s}{s_{3}}\right)+1+\right. \\
& +\frac{s}{s_{3}}\left(2 \ln ^{2}\left(\frac{s}{s_{3}}\right)+10 \ln \left(\frac{s}{s_{3}}\right)+4+\frac{2}{3} \pi^{2}\right)+ \\
& \left.+O\left(\left(\frac{s}{s_{3}}\right)^{2}\right)\right], \\
& G_{2}\left(s, s, s_{1}\right) \stackrel{s \lll s_{1}}{=}-\frac{4}{s s_{1}}\left[\ln \left(\frac{s}{s_{1}}\right)+\right. \\
& +\frac{s}{s_{1}}\left(2 \ln ^{2}\left(\frac{s}{s_{1}}\right)+8 \ln \left(\frac{s}{s_{1}}\right)+2+\frac{2}{3} \pi^{2}\right)+ \\
& \left.+O\left(\left(\frac{s}{s_{1}}\right)^{2}\right)\right] .
\end{aligned}
$$

These asymptotics have to play important role in constraining the effective nonperturbative models when calculating the loops of dynamical quarks $[13$.

In some recent works, however, it was claimed and used a so-called "new QCD constraint" on the pion exchange LbL contribution to the muon $g-2$ [9,16,10]. This "constraint" is an attempt to restrict the behavior of the piontwo-photon vertex when the pion is off-shell. In particular, in [9] it was claimed that it would be followed from QCD that

$$
\mathrm{F}_{\pi^{*} \gamma^{*} \gamma}^{\mathrm{oLMDV}}\left(q^{2} ; q^{2}, 0\right) \stackrel{q^{2} \gg \Lambda_{\mathrm{QCD}}^{2}}{=} \frac{1}{3} f_{0} \chi
$$

where $f_{0}$ is the pion-decay constant in the chiral limit and $\chi$ is the quark condensate magnetic susceptibility in the presence of a constant external electromagnetic field. Let us show that (37) is inconsistent with OPE and QCD constraints (29)- (34).

Indeed, in 9 the off-shell pion form factor is constructed as (off-shell LMD $+\mathrm{V}$ model)

$$
\begin{aligned}
& \mathrm{F}_{\pi^{*} \gamma^{*} \gamma^{*}}^{\mathrm{oLMD}}\left(q_{3}^{2} ; q_{1}^{2}, q_{2}^{2}\right)= \\
& \quad=\frac{f_{\pi}}{3} \frac{-q_{1}^{2} q_{2}^{2}\left(q_{1}^{2}+q_{2}^{2}+q_{3}^{2}\right)+P_{H}^{V}\left(q_{3}^{2} ; q_{1}^{2}, q_{2}^{2}\right)}{\left(q_{1}^{2}+M_{1}^{2}\right)\left(q_{1}^{2}+M_{2}^{2}\right)\left(q_{2}^{2}+M_{1}^{2}\right)\left(q_{2}^{2}+M_{2}^{2}\right)} \\
& P_{H}^{V}\left(q_{3}^{2} ; q_{1}^{2}, q_{2}^{2}\right)=h_{1}\left(q_{1}^{2}+q_{2}^{2}\right)^{2}+h_{2} q_{1}^{2} q_{2}^{2}+ \\
& \quad+h_{3}\left(q_{1}^{2}+q_{2}^{2}\right) q_{3}^{2}+h_{4} q_{3}^{4}-h_{5}\left(q_{1}^{2}+q_{2}^{2}\right)-h_{6} q_{3}^{2}+h_{7},
\end{aligned}
$$

where $M_{(1,2)}$ are the the masses of the lowest vector meson states, and the coefficients in (38) are fixed from the above mentioned constraints. Specifically, $h_{7}$ is due to the pion two-photon decay width, $h_{1}=0$ by requirement (25), $h_{5}$ is from the fit of the CLEO data 29], $h_{2}$ is related to higher power corrections in (25) and finally $\left(h_{3}+h_{4}\right) M_{1}^{-2} M_{2}^{-2}=\chi$ is fixed from the "new constraint" (37). As a result from (38) one get the asymptotics of the pion-exchange part of the total LbL amplitude as

$$
\begin{aligned}
& G_{2, P S}^{\mathrm{oLMDV}}\left(q_{3}^{2}, q_{2}^{2}, q_{1}^{2}\right)= \\
& \quad=\frac{\mathrm{F}_{\pi^{*} \gamma^{*} \gamma^{*}}\left(q_{3}^{2} ; q_{1}^{2}, q_{2}^{2}\right) \mathrm{F}_{\pi^{*} \gamma^{*} \gamma}\left(q_{3}^{2} ; q_{3}^{2}, 0\right)}{q_{3}^{2}+M_{\pi}^{2}}, \\
& G_{2, P S}^{\mathrm{oLMDV}}(s, s, s) \stackrel{s \gg \Lambda_{\mathrm{QCD}}^{2}}{=}-\frac{1}{3} f_{0}^{2} \chi \frac{1}{s^{2}}, \\
& G_{2, P S}^{\mathrm{oLMDV}}\left(s_{3}, s, s\right) \stackrel{s \gg s_{3} \gg \Lambda_{\mathrm{QCD}}^{2}}{=}-\frac{2}{9} f_{0}^{2} \chi \frac{1}{s s_{3}},
\end{aligned}
$$

Comparing the asymptotic coefficients in (40) and (41) with corresponding QCD coefficients in (29) and (33) it is clear that the results based on the "constraint" (37) are in contradiction with the QCD asymptotics obtained in a model independent way. We have already commented in 13 on the origin of this confusion as an incorrect identification of the $\langle P V V\rangle$ correlator with $\langle\pi V V\rangle$. In the latter case, it appears the physical hadronic pseudoscalar current that leads to much higher suppression (42) than it is shown in (37) where the local pseudoscalar current is used. The behavior (37) is in variance with the prediction of the $\mathrm{N} \chi \mathrm{QM}$ where from (18) one has at large $q^{2}$

$$
\mathrm{F}_{P^{*} \gamma^{*} \gamma}^{\mathrm{N} \chi \mathrm{QM}}\left(q^{2} ; q^{2}, 0\right) \stackrel{q^{2} \gg \Lambda_{\mathrm{QCD}}^{2}}{\sim} \exp \left(-q^{2} / \Lambda^{2}\right)
$$

that does not violate the OPE results.

At this point, for completeness, we remind that the $\mathrm{LMD}+\mathrm{V}$ model [7] corresponds to the expression (38) at $q_{3}^{2}=0$. The VMD model, used in particular in 7 to describe the form factors of the $\eta$ and $\eta^{\prime}$ mesons, is defined as

$$
\mathrm{F}_{P \gamma^{*} \gamma^{*}}^{\mathrm{VMD}}\left(0 ; q_{1}^{2}, q_{2}^{2}\right)=\frac{g_{P \gamma \gamma} \Lambda_{M}^{4}}{\left(q_{1}^{2}+\Lambda_{M}^{2}\right)\left(q_{2}^{2}+\Lambda_{M}^{2}\right)},
$$

where the parameters $\Lambda_{M}$ are taken from the fit of CLEO data [29]: $\Lambda_{\pi}=776 \pm 22 \mathrm{MeV}, \Lambda_{\eta}=774 \pm 29 \mathrm{MeV}$, $\Lambda_{\eta^{\prime}}=859 \pm 28 \mathrm{MeV}$.

As a final remark we note, that from the results of 37 . 8, 38, it is easy to get the correct expression that includes the magnetic susceptibility $\chi$ in the asymptotic expansion of the total light-by-light amplitude. Indeed, by using the OPE as it was shown in [37] (see also 38]) due to spontaneous breaking of the chiral symmetry there is the power correction to $w_{L}\left(q^{2}\right)$ that is linear in the current quark mass

$$
\Delta w_{L}\left(q^{2}\right)=\frac{16 \pi^{2}}{3} \frac{m_{c}|\langle\bar{q} q\rangle| \chi}{q^{4}} .
$$

Then, we have the power correction to the total light-bylight amplitude

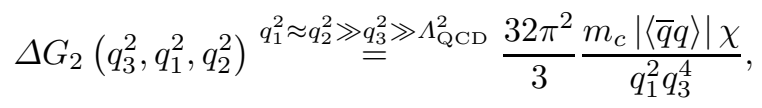

which is suppressed by the current quark mass and by extra power of large momentum squared.

Thus our conclusion is that the effective models, in particular the instanton liquid based $\mathrm{N} \chi \mathrm{QM}$ model, are 
able to satisfy to most of phenomenological and QCD constraints and take into account the full kinematic dependence on virtualities of the mesons and photons. As to VMD based models, it is the LMD+V model [7] for the pion form factor that satisfies to many constraints. The most serious problem of the VMD models is that they do not take into account the dependence of the meson-twophoton vertex on the meson virtuality and thus the momentum conservation is violated in this vertex. We discuss this point with more details in the next section.

\section{Results and comparison with other models}

The results of different approaches in calculation of the pseudoscalar meson contributions to the muon AMM from light-by-light scattering mechanism are given in Table 4. For the pion contribution to the muon AMM in $\mathrm{N} \chi \mathrm{QM}$ we take the numbers from the $S U(2)$ version of the model. For $\eta$ and $\eta^{\prime}$ we use the average of the results obtained with different parameter sets, see Table 3 .

One can conclude that within the $\mathrm{N} \chi \mathrm{QM}$ the pseudoscalar meson contributions to muon AMM are systematically lower then the results obtained in the other works. This effect can be understood considering more carefully the off-shell meson-two-photon vertex $F_{P^{*} \gamma^{*} \gamma}\left(p^{2} ; p^{2}, 0\right)$ entering the light-by-light contribution Eq. (20). The comparison of the $\mathrm{N} \chi \mathrm{QM}$ predictions for the pion in this kinematics (18) with other models is presented in Fig. 6. One can see that the $\mathrm{N} \chi \mathrm{QM}$ leads to stronger suppression of the vertex at all momenta. This explains why the results

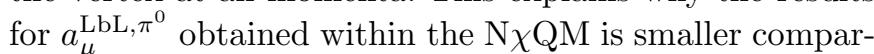
ing with other calculations.

The situation with $\eta^{*}$ s is more dramatic. For illustration in Fig. 7 we present on the same plot the vertex $F_{P^{*} \gamma \gamma}\left(p^{2} ; 0,0\right)$ in the timelike region $p^{2} \leq 0$ and the vertex $F_{P^{*} \gamma^{*} \gamma}\left(p^{2} ; p^{2}, 0\right)$ in the spacelike region $p^{2} \geq 0$ as they look in the $\mathrm{N} \chi \mathrm{QM}$ and VMD models. These two special kinematics match at zero virtuality $p^{2}=0$. The remarkable feature of this construction is that the first kinematics is connected with the decay of pseudoscalar mesons into two photons at physical points $F_{P \gamma \gamma}\left(-M_{M}^{2} ; 0,0\right)=g_{P \gamma \gamma}$, while the second kinematics is relevant for the light-bylight contribution to the muon AMM (20). Thus, the part of Fig. (7) at $p^{2}<0$ describes the transition of the pion-twophoton vertex from the physical points of meson masses to the point with zero virtuality, which is the edge point of the interval where the integrand of (20) is defined. In VMD type of models, including LMD $+\mathrm{V}$ model, there is no such dependence on the meson virtuality. Thus, the value of this vertex at zero meson virtuality is the same as the value of the vertex at the physical points of meson masses, $F_{P \gamma \gamma}^{\mathrm{VMD}}\left(p^{2}=-M_{\eta, \eta^{\prime}}^{2} ; 0,0\right)=F_{P \gamma \gamma}^{\mathrm{VMD}}(0 ; 0,0)$. However, the $\eta$ and $\eta^{\prime}$ mesons are much heavier than the pion and such extrapolation is too crude. One can see that for $\eta$ and particularly for $\eta^{\prime}$ the difference between the values of the vertex at physical and zero virtuality points is large in the N $\chi \mathrm{QM}, F_{P \gamma \gamma}\left(p^{2}=-M_{\eta, \eta^{\prime}}^{2} ; 0,0\right) \gg F_{P \gamma \gamma}(0 ; 0,0)$. Thus, the contributions of the $\eta$ and $\eta^{\prime}$ mesons to the muon AMM evaluated in $\mathrm{N} \chi \mathrm{QM}$ are strongly suppressed as compared with the VMD results that can only be considered as upper estimates of these contribution: 7 .

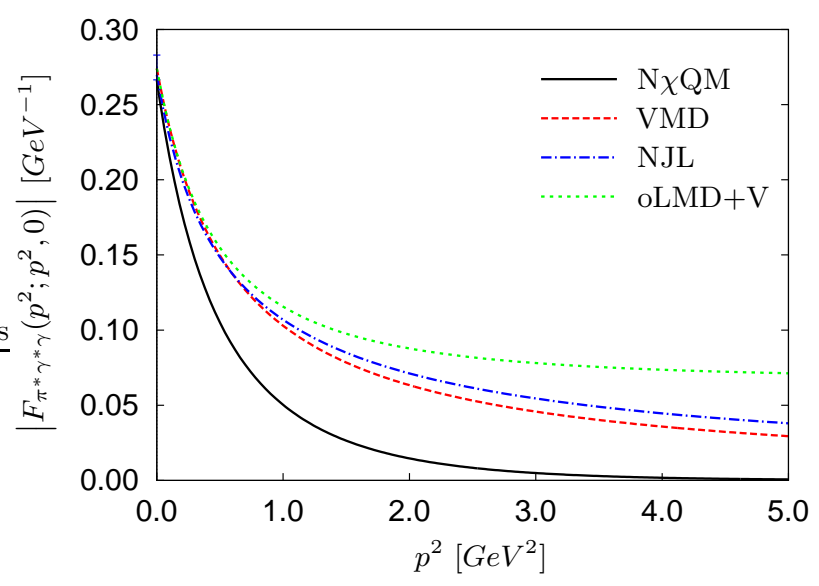

Fig. 6. The photon-pion vertex for the special kinematics when the pion and one photon are off-shell and have the same momenta, and another photon is real. The N $\chi$ QM, Eq. (18), is given by the solid line, the VMD, Eq. (43), is by the dashed line, the NJL result 12 is by dash-dotted line, and oLMD $+\mathrm{V}$, Eq. (38), is by the short dashed line.

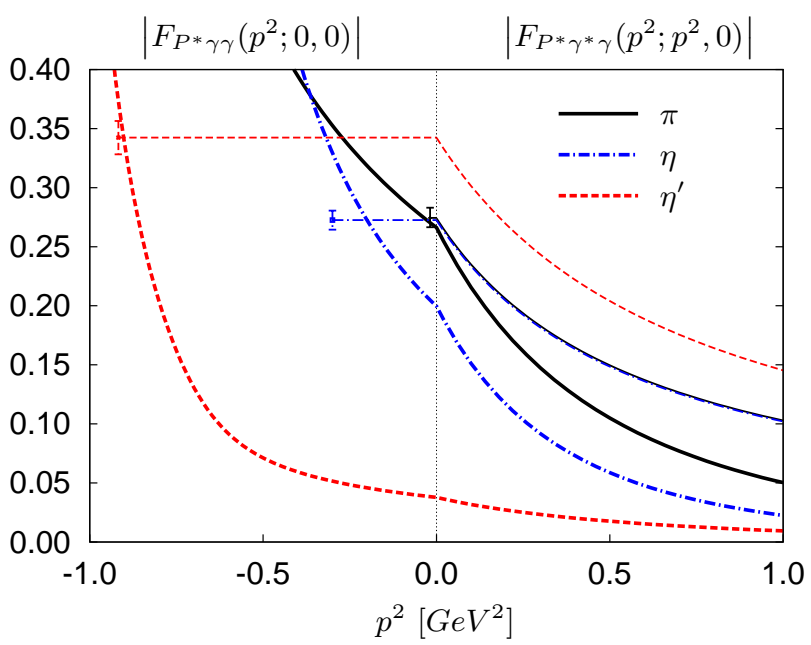

Fig. 7. Plots of the $\pi^{0}, \eta$ and $\eta^{\prime}$ vertices $F_{P^{*} \gamma \gamma}\left(p^{2} ; 0,0\right)$ in the timelike region and $F_{P^{*} \gamma^{*} \gamma}\left(p^{2} ; p^{2}, 0\right)$ in the spacelike region in $\mathrm{N} \chi \mathrm{QM}$ model (thick lines) and VMD, Eq. (43), model (thin lines). The points with error bars correspond to the physical points of the meson decays into two photons. The VMD curves for $\pi^{0}$ and $\eta$ are almost indistinguishable.

\footnotetext{
7 Recently another attempt to take into account the full kinematic dependence of the pion-photon vertex is given in the DSE approach [15. In this paper, the meson's contribution is investigated using the pion-pole approximation with an off-shell prescription. Although this prescription leads to a small suppression a full T-matrix calculation therein is highly desirable. We thank Richard Williams for clarifying this point.
} 


\begin{tabular}{|c|c|c|c|c|}
\hline Model & $\pi^{0}$ & $\eta$ & $\eta^{\prime}$ & $\pi^{0}+\eta+\eta^{\prime}$ \\
\hline VMD [6] & 5.74 & 1.34 & 1.19 & $8.27(0.64)$ \\
\hline ENJL [1] & 5.6 & & & $8.5(1.3)$ \\
\hline $\mathrm{LMD}+\mathrm{V}, \mathrm{VMD}[7]$ & $5.8(1.0)$ & $1.3(0.1)$ & $1.2(0.1)$ & $8.3(1.2)$ \\
\hline NJL 12 & $8.18(1.65)$ & $0.56(0.13)$ & $0.80(0.17)$ & $9.55(1.66)$ \\
\hline$(\mathrm{LMD}+\mathrm{V})^{\prime}, \mathrm{VMD} 8$ & 7.97 & 1.8 & 1.8 & $11.6(1.0)$ \\
\hline $\mathrm{N} \chi \mathrm{QM} 13$ & $6.5(0.2)$ & & & \\
\hline HM 16] & 6.9 & 2.7 & 1.1 & 10.7 \\
\hline DIP, VMD 10 & $6.54(0.25)$ & & & \\
\hline DSE 15 & $5.75(0.69)$ & $1.36(0.30)$ & $0.96(0.21)$ & $8.07(1.20)$ \\
\hline This work $(\mathrm{N} \chi \mathrm{QM})$ & $5.01(0.37)$ & 0.54 & 0.30 & 5.85 \\
\hline
\end{tabular}

Table 4. The light-by-light contribution to the muon AMM from the hadronic exchanges in the neutral pseudoscalar channel $a_{\mu}^{\mathrm{LbL}}$ obtained in different works. All numbers are given in $10^{-10}$. Note, that as it was checked in [41, 13, the (LMD+L)' model [8] numerically predicts 7.97 instead of 7.65 quoted originally in 8 .

\section{Conclusions}

In this paper we have analyzed the pseudoscalar meson exchange contributions of the light-by-light process to $a_{\mu}^{\mathrm{PS}, \mathrm{LbL}}$ in the nonlocal chiral quark model. The basic new elements of our work are the inclusion of the full kinematic dependence of the pseudoscalar meson-photon vertex on virtualities of photons and mesons and the effect of current quark masses. This study extends the previous work [13. where the full kinematic dependence was considered for the pion in the chiral limit. The dependence of the vertices on the pion virtuality diminishes the result by about $20-30 \%$ as compared to the case where this dependence is neglected. We find that the most significant effect occurs for the contributions of the $\eta$ and $\eta^{\prime}$ mesons to the muon AMM. In this case the results are reduced by factor about 3 in comparison with the results obtained in other effective quark models where the kinematic dependence was neglected. Thus our main conclusion is that within the realistic nonlocal chiral quark model the total contribution of pseudoscalar exchanges to $a_{\mu}^{\mathrm{PS}, \mathrm{LbL}}$ is by approximately factor 1.5 less than the most of previous estimates.

We reviewed the phenomenological and QCD constraints on the hadronic light-by-light scattering amplitude. It is shown that the effective models like $\mathrm{N} \chi \mathrm{QM}$ are able to fit most of these constraints, while the models based on the vector meson dominance violate the momentum conservation law and lead to overestimation of the meson exchanges contribution to the muon AMM. We have also demonstrated that one of the constraints for the LbL amplitude recently discussed in the literature [9] is not justified.

An important next step in the investigations of the light-by-light scattering within the nonlocal chiral quark model is to perform an extension to the so-called complete calculation (see $42,6,12$ ) that includes the scalar and axial-vector meson exchanges, as well as takes into account the quark and meson box diagrams. Due to contact terms arising in the nonlocal model, a calculation of the box diagrams is technically rather involved and will be presented elsewhere.
This work is supported in part by the program Development of Scientific Potential in Higher Schools (2.2.1.1/1483, 2.1.1/1539), the Federal Target Program "Research and Training Specialists in Innovative Russia 2009-2013" (02. 740.11.5154), JINRBelarus project N183, RBFR grants 09-02-00749, 10-02-00368 and 11-02-00112.

\section{References}

1. G.W. Bennett et al. [Muon (g-2) Collaboration], Phys. Rev. D 73 (2006) 072003.

2. F. Jegerlehner and R. Szafron, arXiv:1101.2872 [hep-ph].

3. M. Davier, A. Hoecker, B. Malaescu and Z. Zhang, Eur. Phys. J. C 71 (2011) 1515.

4. M. Hayakawa, T. Kinoshita and A.I. Sanda, Phys. Rev. Lett. 75 (1995) 790.

5. J. Bijnens, E. Pallante and J. Prades, Phys. Rev. Lett. 75 (1995) 1447.

6. M. Hayakawa and T. Kinoshita, Phys. Rev. D 57 (1998) 465.

7. M. Knecht and A. Nyffeler, Phys. Rev. D 65 (2002) 073034.

8. K. Melnikov and A. Vainshtein, Phys. Rev. D 70 (2004) 113006

9. A. Nyffeler, Phys. Rev. D 79 (2009) 073012.

10. L. Cappiello, O. Cata and G. D'Ambrosio, arXiv:1009.1161 [hep-ph].

11. J. Bijnens, E. Pallante and J. Prades, Nucl. Phys. B 626 (2002) 410.

12. E. Bartos, A. Z. Dubnickova, S. Dubnicka, E. A. Kuraev and E. Zemlyanaya, Nucl. Phys. B 632 (2002) 330.

13. A. E. Dorokhov and W. Broniowski, Phys. Rev. D 78 (2008) 073011.

14. C. S. Fischer, T. Goecke and R. Williams, arXiv:1009.5297 [hep-ph].

15. T. Goecke, C. S. Fischer and R. Williams, arXiv:1012.3886 [hep-ph].

16. D. K. Hong and D. Kim, Phys. Lett. B 680 (2009) 480.

17. E. de Rafael, Phys. Lett. B 322 (1994) 239.

18. E. Pallante, Phys. Lett. B 341 (1994) 221.

19. B. Holdom, R. Lewis and R.R. Mendel, Z. Phys. C 63 (1994) 71.

20. A.A. Pivovarov, Phys. Atom. Nucl. 66 (2003) 902 [Yad. Fiz. 66 (2003) 934].

21. A.E. Dorokhov, Phys. Rev. D 70 (2004) 094011. 
22. I.V. Anikin, A.E. Dorokhov and L. Tomio, Phys. Part. Nucl. 31 (2000) 509 [Fiz. Elem. Chast. Atom. Yadra 31 (2000) 1023].

23. A.E. Dorokhov and W. Broniowski, Eur. Phys. J. C 32 (2003) 79 .

24. A. Scarpettini, D. Gomez Dumm and N. N. Scoccola, Phys. Rev. D 69 (2004) 114018.

25. A. E. Dorokhov, JETP Lett. 77 (2003) 63 [Pisma Zh. Eksp. Teor. Fiz. 77 (2003) 68].

26. F. Jegerlehner and A. Nyffeler, Phys. Rept. 477 (2009) 1.

27. K. Nakamura et al. [Particle Data Group], J. Phys. G 37, 075021 (2010).

28. H. J. Behrend et al. [CELLO Collaboration], Z. Phys. C 49 (1991) 401.

29. J. Gronberg et al. [CLEO Collaboration], Phys. Rev. D 57 (1998) 33.

30. B. Aubert et al. [BABAR Collaboration], Phys. Rev. D 80 (2009) 052002.

31. P. del Amo Sanchez et al. [BABAR Collaboration], arXiv:1101.1142 [hep-ex].

32. A. Nyffeler, PoS C D09 (2009) 080.

33. A. E. Dorokhov, Phys. Part. Nucl. Lett. 7 (2010) 229-234.

34. A.E. Dorokhov, JETP Lett. 91 (2010) 163 [Pisma Zh. Eksp. Teor. Fiz. 91 (2010) 175].

35. S.J. Brodsky and G.P. Lepage, Phys. Rev. D 24 (1981) 1808.

36. V.A. Novikov, M.A. Shifman, A.I. Vainshtein, M.B. Voloshin and V.I. Zakharov, Nucl. Phys. B 237 (1984) 525 .

37. A. Vainshtein, Phys. Lett. B 569, 187 (2003).

38. A.E. Dorokhov, Eur. Phys. J. C 42 (2005) 309.

39. A.E. Dorokhov, JETP Lett. 82 (2005) 1 [Pisma Zh. Eksp. Teor. Fiz. 82 (2005) 3].

40. A.I. Davydychev and J.B. Tausk, Phys. Rev. D 53 (1996) 7381.

41. J. Bijnens and J. Prades, Mod. Phys. Lett. A 22 (2007) 767.

42. J. Bijnens, E. Pallante and J. Prades, Nucl. Phys. B 474 , 379 (1996). 